\title{
El problema mente-cuerpo y el dualismo naturalista: bases para considerar rechazar un acercamiento naturalista y reconsiderar seriamente un acercamiento fenomenológico al problema de la conciencia
}

Emily Noejovich

Pontificia Universidad Católica del Perú

Resumen: El presente trabajo consiste en un breve análisis hermenéutico de los conceptos principales en los que se sostiene la interpretación de Chalmers del problema mente-cuerpo. Nuestra reflexión apunta a criticar la postura de Chalmers y dado que la estructura de esta interpretación es reflejo de una tradición filosófica; esto es, reproduce un tipo de aproximación, postulamos que la inefectividad de su planteamiento es razón suficiente para reconsiderar otro tipo de acercamiento frente al mismo problema: la aproximación proveniente de la tradición fenomenológica.

Palabras clave: experiencia consciente, naturalismo, dualismo, mente-cuerpo, fenomenología

Abstract: The present paper aims at setting an hermeneutic analysis of the main concepts Chalmers' interpretation on the mind-body problem is built upon. Our reflection aims at criticizing Chalmers' position and given that the structure of his interpretation reflects a philosophical tradition; that is, it reproduces a type of approach, we believe that the ineffectiveness of his approach is reason enough to reconsider another type of approach towards the same problem: the one provided by the phenomenological tradition.

Key words: conscious experience, naturalism, dualism, mind-body, phenomenology 


\section{§1. Introducción: el hiato cartesiano mente - cuerpo}

De manera patente, desde Descartes el mundo occidental se ha visto perseguido por el problema que abre la postura dualista cartesiana en torno a la relación entre lo mental (res cogitans) y lo corporal (res extensa), el cual ha adquirido diversas formas de expresarse, así como también ha tomado diversos nombres para ser identificado. Es así que para referirnos a él podemos bien hablar del problema de la reconciliación entre sujeto y objeto, y, consecuentemente, entre el ámbito de lo subjetivo y de lo objetivo. Asimismo, podemos hablar más simplemente del problema mente-cuerpo, el cual es, en nuestros tiempos, la manera anglosajona característica de clasificarlo, tematizarlo y, en suma, de referirse a él.

Nos permitimos hacer este hincapié, pues si bien el hablar de un "problema mente-cuerpo" no es ninguna novedad ni originalidad histórica, ni de una designación exclusiva del horizonte filosófico anglosajón, pareciera a veces, por la literatura de sus distintos exponentes, que el privilegiar esta forma de hablar acerca del problema es una manera de comprenderlo que se permite obviar este otro aspecto: el del sujeto/objeto (lo que hemos denominamos más arriba como "la reconciliación entre sujeto y objeto"). Creemos que este acto de obviar deviene posteriormente ya no solo un permiso para no tomarlo en directa consideración, sino una negación de tal aspecto, el cual, no obstante, creemos crucial para la superación del problema en cuestión. Así, consideramos como ejemplos pertinentes de lo anterior a las posturas reductivas o eliminativistas tales como la de Daniel Dennett o, incluso, dentro de las posturas funcionalistas cercanas a aproximaciones fenomenológicas ${ }^{1}$, la misma postura de David Chalmers, la cual será para nosotros de especial interés. Tal es así que la intención que nos convoca es discutir el fundamento en el que descansa la postura de Chalmers en relación a la observación que hemos brevemente esbozado acá; esto es, en tanto lectura (interpretación, diagnóstico y prescripción) del problema.

1 Estamos siguiendo la clasificación que esboza Francisco Varela en Neurophenomenology: A Methodological Remedy for the Hard Problem (1996). Ahí, Varela identifica la postura de Chalmers como cercana a una aproximación fenomenológica en la medida en que existe en ella, al menos, un reconocimiento de la irreductibilidad de la naturaleza de la experiencia consciente (1998 [1996]: 340). 
Asimismo, pensamos que, aunque cada manera de referirnos al problema implica señalar un aspecto determinado del mismo, el problema sigue siendo el mismo. Este radica en el hecho de que, a partir de Descartes, se instaura un hiato o escisión entre lo mental (la mente) y lo físico (el cuerpo) que polariza estas dimensiones partiendo de la tesis de que son de naturalezas distintas. De este modo, la naturaleza del problema es de origen metafísico (ontológico) ${ }^{2}$ y acarrea consecuencias epistemológicas en la medida en que deja abierta la pregunta por cómo interactúan ámbitos (o realidades) tan distintos. Y si bien ha transcurrido bastante tiempo desde Descartes, la interpretación dualista del problema mente-cuerpo de corte metafísico ${ }^{3}$ dista mucho de haber sido enterrado. Un ejemplo de ello es la postura de David Chalmers, filósofo anglosajón contemporáneo, quien defiende una versión actualizada del dualismo cartesiano a la cual denomina dualismo naturalista. De ahí surge nuestro interés por este autor.

Así pues, la finalidad del presente trabajo es, de manera general, examinar la postura de Chalmers en torno al problema mente-cuerpo. Y, de manera específica, nuestro objetivo es esbozar una crítica a lo que postula Chalmers como superación de dicho problema. Dicho de manera breve, la tesis de Chalmers sostiene que para superar el problema del hiato mente-cuerpo, "(...) los métodos usuales de explicación de la ciencia cognitiva y de la neurociencia no son suficientes (...)"4 (Chalmers 1998a: 135); de modo que "necesitamos un ingrediente extra en la explicación" (Chalmers, 1998a: 17). En última instancia, esto quiere decir que la superación estriba en que

2 Al menos en Descartes, la tesis de la dualidad mente-cuerpo es una tesis que sostiene la existencia de dos sustancias de naturalezas distintas: res cogitans y res extensa. Si bien la naturaleza del origen del problema mente-cuerpo, como hemos dicho, es metafísica, no se mantiene necesariamente así. Hoy en día existe una gran variedad de posiciones frente a este problema que no lo identifican (o, al menos, intentan no identificarlo) como un problema metafísico, pero la constante ( $y$, por tanto, lo importante) es que se sostiene que hay dos dominios (realms) cuya interacción es vista como un problema.

3 Asumiremos los términos "metafísico" y "ontológico" como equivalentes.

4 Todos los textos de Chalmers están escritos originalmente en inglés. La traducción al castellano de todos los textos de Chalmers que citamos es siempre nuestra. Las traducciones de otros textos escritos originalmente en inglés tales como los de Varela, Gallagher y Zahavi, serán también nuestras.

5 El texto de Facing Up to the Problem of Consciousness es originalmente del año 1995. Cuando citamos este artículo, lo hacemos desde la recopilación que hace Shear Explaining Consciousness: The Hard Problem publicada en 1998. 
tomemos "(...) a la experiencia misma como una característica (feature) fundamental del mundo, al lado de la masa, la carga [electromagnética] y el espacio-tiempo" (Chalmers, 1998a: 20); esto es, que se introduzca una modificación (por expansión en este caso) de la ontología con la que parte la ciencia, tal y como sucedió con el electromagnetismo en su tiempo.

Por ejemplo, en el siglo XIX resultó que los procesos electromagnéticos no podían explicarse en términos de los procesos totalmente mecánicos a los que recurrían las teorías físicas anteriores, Maxwell y otros introdujeron la carga electromagnética y las fuerzas electromagnéticas como nuevos componentes fundamentales de una teoría física. Para explicar el electromagnetismo, había que explicar la ontología de la física (Chalmers, 1998a: 19).

Por nuestro lado, la tesis de la que parte nuestra crítica es que la superación del hiato mente-cuerpo se encuentra en leer el problema como el de la conciliación entre el sujeto y el objeto, donde el primero se considera el agente que constituye al segundo. De esta manera, la superación se entendería, más bien, como dar metafóricamente un giro hacia nuestra experiencia misma (esto es, examinar aquello que se nos presenta como lo más obvio, natural y cercano); la superación pasaría, en términos de Varela, en “la exploración sistemática del único vínculo entre la mente y la conciencia (...) [el cual radica en] la estructura de la experiencia humana misma" (Varela, 1998: 337, cursivas añadidas). Y esto implica, en el trasfondo, circunscribirnos a una lectura más cercana a la fenomenología ${ }^{6}$ como forma de superar el hiato. De este modo, sostenemos que la superación en cuestión no debería ni tendría por qué seguir un camino ontológico y conducirnos a postular una nueva entidad fundamental a partir de la cual derivar principios que permitan esbozar una teoría unificadora, sino que podría encontrarse, más bien, en el más modesto camino de cambiar nuestra perspectiva frente a lo que está en juego en el problema mismo.

6 Nos permitimos hablar de fenomenología en general, pues no es el objetivo central de este trabajo desarrollar a cabalidad esta tesis nuestra por la gran extensión que ello requeriría. Reconocemos, así, la gran complejidad del tema y las limitaciones que actualmente nos restringen para su cabal elaboración. No obstante, cuando decimos que la superación del hiato mente-cuerpo implicaría tomar un camino más afín a la fenomenología estamos pensando, principalmente, en la fenomenología de Husserl. Sin embargo, también tenemos en mente la reactualización (en cierta medida) de la fenomenología husserliana por parte de filósofos contemporáneos como Dan Zahavi y Shaun Gallagher, y de científicos como Francisco Varela. 
Nuestro trabajo se dividirá en tres partes principales. En primer lugar, examinaremos a fondo el dualismo naturalista que defiende David Chalmers. Esta parte, a su vez, se dividirá en dos secciones: la primera está dedicada a caracterizar la interpretación del problema mente-cuerpo desde la postura dualista naturalista, mientras que la segunda se ocupará de caracterizar la superación de dicho problema que plantea tal postura. En segundo lugar, nos dedicaremos a esbozar, a modo de crítica, los problemas que encontramos en el dualismo naturalista. Estos tienen que ver, de un lado, con las implicancias ontológicas con las que se compromete el dualismo naturalista (la posibilidad del panpsiquismo) y, de otro, con los presupuestos en los que se funda el dualismo naturalista (los cuales, a su vez, posibilitan estas ulteriores implicancias ontológicas). La crítica común a ambos problemas radica en que el concepto principal de tal postura no cumple un rol explicativo. Finalmente, en la última parte, realizaremos una síntesis de los puntos importantes y elaboraremos una reflexión final que delinee y esclarezca nuestra propia posición, la cual subyace a lo largo de las dos primeras partes.

\section{§2. David Chalmers y el dualismo naturalista}

Como ya anticipamos, Chalmers denomina a su postura dualismo naturalista. Por un lado, su caracterización dualista refiere al hecho de que "postula propiedades básicas sobre y por encima de las propiedades invocadas por la física" (Chalmers, 1998a: 20). Se trata, así, de un dualismo de clara herencia cartesiana, pero cuyo punto de inflexión estriba en que no postula dos sustancias de naturalezas distintas (res cogitans versus res extensa) que compongan la realidad, sino en postular que esta, la realidad, consta de dos tipos de propiedades distintas: el tipo físico y el tipo mental ${ }^{7}$. De esta manera, nada en su aproximación contradice lo ya asentado por la física (Chalmers, 1998a: 20) y es, por lo tanto, compatible con la mirada científica.

$7 \quad$ Estrictamente, este es el tipo de propiedad al que denomina fenomenal. Se trata del fenómeno sensible entendido en oposición a lo físico medible. Chalmers llega a esta denominación, luego de realizar un análisis respecto de los dos sentidos que cree que componen el concepto de mente. 
La ciencia puede entenderse, entonces, como un corpus explicativo que parte de entidades fundamentales que son gobernadas por leyes y a partir de las cuales se derivan teorías: “(...) las entidades fundamentales no son explicadas en términos de nada más simple. En su lugar, uno las toma como básicas y brinda una teoría de cómo se relacionan con todo lo demás en el mundo" (Chalmers, 1998a: 19). Las entidades fundamentales que acepta la ciencia (la física especialmente), de acuerdo con Chalmers, incluyen la masa, el espacio-tiempo y el electromagnetismo ${ }^{8}$, y todas estas comparten el ser instancias de un mismo tipo de propiedad; esto es, el tipo físico. Con esto notamos desde ya que su intención, en lo que refiere a la superación del problema mente-cuerpo, apunta a sostener que lo mental es un tipo de propiedad que se instancia en una entidad fundamental": esta entidad es lo que él denominará "experiencia consciente", "experiencia" o "conciencia" (consciousness), entre otras denominaciones posibles de las cuales hablaremos más adelante.

Así pues, Chalmers parte de que la ciencia brinda ya una descripción acertada de la realidad y es esto lo que determina su aproximación y consecuente solución del problema. En razón de lo anterior, describe su postura como “(...) enteramente naturalista (...) el universo en última instancia se reduce (comes down) a una red de entidades básicas que obedecen leyes simples (...) [lo cual permite que] haya en última instancia una teoría de la conciencia dada en términos de tales leyes" (Chalmers, 1998a: 20). Desde su perspectiva naturalista, “(...) la experiencia consciente es un fenómeno natural [y esto significa que] ella es parte del mundo natural y que, como todo otro fenómeno natural, grita por una explicación" (Chalmers, 1998a: 4-5) en ese mismo registro. Dicho en otras palabras, la aproximación de Chalmers al problema sigue una línea metafísica animada y alimentada por el esquema planteado por las ciencias naturales.

8 En Facing Up to the Problem of Consiousness (1995) solo hace referencia a estas tres entidades como entidades fundamentales, pero en Panpsychism and Panprotopsychism (2013) la mención abarca a los quarks y a los fotones.

9 Definición de entidad fundamental: una entidad que no se puede explicar apelando a elementos más pequeños y que es, en ese sentido, irreductible. Este es el sentido que asumiremos y sobre el cual discutiremos en el presente trabajo. 


\section{§2.1. Caracterización del problema mente-cuerpo de acuerdo al dualismo naturalista de Chalmers}

Antes de continuar, debemos esclarecer los siguientes dos puntos. En primer lugar, para Chalmers, aquello que instancia propiedades físicas es aquello que puede ser explicado mecánicamente. Por ejemplo, si se quiere explicar Q y Q es una instanciación de la propiedad del tipo físico, entonces explicar $Q$ es equivalente a explicar cómo $Q$ desempeña una función $F^{10}$. Por su parte, para explicar cómo $Q$ desempeña una función $F$ “(...) solo se necesita especificar un mecanismo que pueda ejecutar la función" (Chalmers, 1998a: 11). De esta manera, al hablar de función nos referimos al rol causal que produce el comportamiento de $Q$ y es en ese sentido que $Q$ es definible funcionalmente (functionally definable); esto es, tal y como sucede en las ecuaciones matemáticas de la forma ??=?. De ahí que aquello que instancia propiedades físicas tenga un carácter objetivo; esto es, en tanto externo (causalmente mediado), medible y mecanizable.

En segundo lugar, Chalmers parte de que hay dos sentidos que componen el concepto de mente: de un lado, un sentido psicológico y, de otro, un sentido fenoménico o fenomenal sensible (fenomenal concept). El sentido psicológico se remite al concepto de la mente "(...) como base causal o explicativa de la conducta (...)" (Chalmers, 1996: 10). En este contexto, "la mente se caracteriza por lo que hace" (Chalmers, 1996: 10). De esta manera, un estado mental $\mathrm{P}$ es un estado psicológico si $\mathrm{P}$ desempeña un papel causal apropiado o bien en la producción misma de la conducta o bien en la explicación de tal conducta (Chalmers, 1996: 10).

Así, incorporando lo dicho anteriormente, notamos que hay para Chalmers una manera, digamos, física de entender la mente, la cual es plasmada en el sentido psicológico. Esto quiere decir que, si un estado mental $\mathrm{P}$ es un estado psicológico, entonces es definible funcionalmente y, por lo tanto, explicable "(...) en términos de mecanismos computacionales o neuronales (...)" (Chalmers, 1998a: 10). Este es justamente el objeto de la ciencia

10 No nos estamos pronunciando aquí en un sentido teleológico; es decir, no estamos afirmando en nuestro ejemplo que F sea la función para la cual P existe. 
cognitiva: las habilidades cognitivas y sus respectivas funciones (en tanto causas que ocasionan determinados efectos; ergo, determinadas conductas). Por consiguiente, la mente, en el sentido psicológico, es vulnerable a las explicaciones mecánicas y reductivas de la ciencia; esto es, a aquellas dedicadas a resolver problemas de ejecución de funciones (performance of functions).

Ahora bien, si la mente para Chalmers se agotara en el sentido psicológico, entonces no habría ningún tipo de dualidad, pues lo mental sería explicable sobre la base de mecanismos que desempeñan funciones específicas que, al ejecutarse, causarían determinadas conductas (lo externo observable). Pero Chalmers sostiene que existe un segundo sentido del concepto de mente: el sentido fenoménico o fenomenal sensible, en el cual fundamenta su dualismo. Para intentar esclarecer, en la medida de lo posible, el significado de esta forma de caracterizar a la mente en tanto fenoménica sensible, hay que tomar en cuenta, en primer lugar, que lo crucial está en que tal concepto no se refiere a la actividad mental como una ejecución de mecanismos, sino que hace referencia a la experiencia misma que acompaña tal ejecución.

En ese sentido, Chalmers distingue el sentido fenoménico sensible como aquel que caracteriza a "la mente por cómo esta se siente para un agente cognitivo" (Chalmers, 1996: 10); es decir, la experiencia misma que acompaña a la ejecución de procesos cognitivos. Tal caracterización conduce a Chalmers a definir el sentido fenoménico sensible en tanto el "(...) concepto de la mente como experiencia consciente y el de un estado mental como estado mental conscientemente experimentado" (Chalmers, 1996: 10). Ahora bien, hay que tener cautela con esta expresión, pues, como pretendemos mostrar en lo que sigue, cuando Chalmers habla de "experiencia consciente" (conscious experience) o simplemente de "experiencia", él no se está refiriendo al movimiento aprehensivo de ningún sujeto, agente, yo o unidad (por decirlo de algún modo), sino simplemente al mero estado cualitativo sensible que caracteriza cómo son las cosas; esto es, como resultado de un proceso cognitivo. 
Al respecto, vale la pena recordar la discusión anterior sobre el sentido psicológico de la mente, pues los procesos cognitivos conforman esta dimensión y, en ese sentido, se entienden siempre como habilidades y funciones reductibles a mecanismos en la medida en que despliegan un rol causal. Así, lo fenoménico sensible radica en el hecho de que la mente, al realizar estos procesos, no solamente lleva a cabo un procesamiento de información, sino que ese procesamiento de información va de la mano con un aspecto cualitativo que define al concepto de experiencia consciente que maneja Chalmers. Tal y como afirma en The Conscious Mind "(...) cuando percibimos, pensamos y actuamos, existe un ruido de fondo de causalidad y procesamiento de información, pero este procesamiento por lo general no ocurre en la oscuridad. Existe también un aspecto interno; hay algo que se siente como siendo [o en tanto que se es] ${ }^{11}$ un agente cognitivo. Este aspecto interno es la experiencia consciente"(Chalmers, 1996: 4).

Dicho en otras palabras, al ver una imagen no solo hay un procesamiento de ondas de radiación electromagnéticas de longitudes específicas, sino que este procesamiento va acompañado de una sensación visual interna; esto es, hay una cualidad subjetiva en la experiencia. Por ejemplo, "(...) el sentir cualitativo [sensible] del rojo, la experiencia de lo oscuro y la luz [o] la cualidad [sensible] de la profundidad en un campo visual" (Chalmers, 1998a: 10). Para Chalmers, decir que hay un aspecto interno significa lo mismo que decir que hay un aspecto subjetivo ${ }^{12}$, y todo ello significa tener una "experiencia consciente" o simplemente "experiencia", en la medida en que se mantiene una misma referencia: la cualidad sensible que acompaña a este procesamiento de información.

Esto es lo que Chalmers denomina "la cualidad subjetiva de la experiencia" (Chalmers, 1995; 1996) y, de manera concomitante, a lo que se refiere

11 Literalmente Chalmers emplea la siguiente expresión: "there is something it feels like to be a cognitive agent" (Chalmers, 1996: 4). Las negritas son nuestras. Solamente queremos resaltar la expresión exacta que usa Chalmers para describir esta situación y otras de este tipo. La traducción al castellano nos parece que no llega a captar la intuitividad de la expresión en inglés.

12 "When we think and perceive, there is a whir of information processing, but there is also a subjective aspect" (Chalmers, 1998a: 10). Colocamos esta cita, pues, si se coteja con la cita anterior, se evidencia la intercambiabilidad de la que estamos hablando. 
cuando dice que "(...) hay algo que se siente como siendo [o en tanto que se es] un agente cognitivo (...)"hay algo que se siente como siendo [o en tanto que se es] un agente cognitivo"13 (Chalmers, 1996: 4). En ese sentido, "un organismo es consciente, si es que existe algo como ser tal organismo (if there is something it is like to be that organism) y un estado mental es consciente si existe algo como estar en tal estado (if there is something it is like to be in that state)" (Chalmers, 1998a: 11). En la medida en que lo anterior resume la caracterización del concepto fenoménico de mente, tal concepto remitiría a la caracterización del modo como (algo) se experimenta ${ }^{14}$. Por lo tanto, en este contexto, el que un estado mental $\mathrm{F}$ sea fenoménico significa que este estado mental F se experimenta de cierto modo; es decir, que tiene una característica cualitativa.

Ahora bien, la distinción entre estos dos sentidos del concepto de mente es crucial, pues implica un modo particular de leer el problema mente-cuerpo. Por un lado, como hemos examinado, el que un estado mental sea psicológico significa que cumple un rol causal. Por lo tanto, en este contexto lo mental se define por lo que hace y ello implica, en consecuencia, que no ofrece ninguna resistencia a una explicación científica: no genera ningún impase a la neurociencia y a las ciencias cognitivas. Consecuentemente, el problema mente-cuerpo no puede ubicarse aquí, en este sentido psicológico de la mente. Y si partimos de que este problema sí existe, este solo podría ubicarse, siguiendo un principio deductivo, en la mente como sentido fenoménico sensible.

Es frente a esta caracterización del problema mente-cuerpo que responde la célebre distinción entre dos tipos de problemas de la conciencia por la cual Chalmers es recordado: la distinción entre los problemas fáciles (the easy problems) y el problema difícil (the hard problem) de la conciencia (consciousness). Lo que Chalmers denomina como los easy problems corresponde al sentido psicológico de la mente, mientras que lo que denomina como el hard problem of consciousness corresponde al concepto fenoménico. El

13 "If there is something it feels like to be a cognitive agent" (Chalmers, 1996: 4).

14 "On the phenomenal concept, mind is characterized by the way it feels" (Chalmers, 1996: $10)$. 
problema mente-cuerpo es, así, sinónimo y exclusivo del hard problem of consciousness.

\section{§2.2. Caracterización de la superación del problema de acuerdo al dualismo naturalista}

En Facing up to the Problem of Consciousness (1995), Chalmers elabora una pequeña lista de aquellos procesos mentales que comprenderían el concepto psicológico de la mente: la habilidad para discriminar, categorizar y reaccionar al estímulo ambiental; la integración de la información por un sistema cognitivo; la reportabilidad de los estados mentales; la habilidad que tiene un sistema para acceder a sus propios estados internos; el enfocar la atención; el control deliberado del comportamiento; y la diferencia entre la vigilia y el sueño. Esta lista aparece sistematizada en The Conscious Mind (1996), de modo que se caracterizan como nociones psicológicas (psychological notions), en el sentido previamente expuesto, las siguientes: vigilia (awakeness), introspección (introspection), reportabilidad o informatividad (reportability), autoconciencia (self-consciousness), atención (attention), control voluntario (voluntary control) y conocimiento (knowledge). Todas son nociones funcionales, lo cual se demuestra por la manera en que pueden responder a la pregunta por cómo uno explicaría el fenómeno en cuestión (Chalmers, 1996: 25).

Si retomamos parte de la discusión previa en torno a lo que entiende Chalmers por aquello que instancia propiedades físicas y lo que caracteriza al sentido psicológico de la mente, el que estos procesos mentales y nociones psicológicas sean clasificados como easy problems of consciousness cae bajo su propio peso: se debe a que todos representan problemas acerca de la ejecución de funciones (performance of functions) y, en ese sentido, para ser resueltos bastaría con encontrar un mecanismo específico que cumpla tal rol. Asimismo, dijimos anteriormente que las nociones psicológicas no ofrecen resistencia a las explicaciones científicas. Esto significa que con la metodología de la ciencia actual y sin la necesidad de asumir un nuevo elemento fundamental, ni nuevos principios, se podría en teoría llegar a una explicación, ya que los métodos de la ciencia "(...) han sido desarrollados 
precisamente para explicar la ejecución de funciones cognitivas (...) [, es decir,] están equipados para explicar la ejecución de funciones (...)" (Chalmers, 1998a: 11). Es por ello que se les denomina métodos reductivos.

Sin embargo, el talón de Aquiles de la explicación científica estriba en que sus métodos solamente están equipados, conceptualmente, para explicaciones de ese tipo. Y, mientras tanto, el problema mente-cuerpo (entendido como el problema de la mente fenoménica sensible o el hard problem of consciousness, tal como lo hemos examinado) no corresponde a un problema relativo al desempeño de funciones. Por consiguiente, se descarta a priori la plausibilidad del éxito del método reductivo de la ciencia para explicar este dominio, ya que la naturaleza misma del sentido fenoménico sensible o de la experiencia consciente (usando el leguaje técnico de Chalmers) imposibilita que la ciencia, empleando sus métodos reductivos, supere el hard problem of consciousness ${ }^{15}$. De ahí que Chalmers se remita al problema caracterizándolo como hard, puesto que ello refleja el hecho de que este sentido de lo consciente (consciousness), el estado cualitativo sensible de la experiencia consciente, genera un impase a la ciencia.

En este panorama, la forma planteada por Chalmers para superar el problema es la siguiente. Chalmers sostiene que debemos admitir una pequeña ampliación en nuestra ontología que sirva de fundamento teórico para una metodología no reductiva. Esto significa que la ciencia dedicada a investigar la mente humana ${ }^{16}$ debe admitir que la realidad no solamente se compone por las entidades fundamentales que la física ha descubierto y que sirven para sostener todas las explicaciones científicas; es decir, que la realidad no solamente está conformada por lo físico, sino también por lo fenoménico sensible de lo mental. En ese sentido, la superación planteada por Chalmers apunta a que debemos considerar a la "experiencia consciente", "experiencia", "conciencia fenomenal" o "qualia" como una entidad fundamental más a la par con las otras entidades fundamentales tales como la masa y el espacio-tiempo, etc. Ello con vistas a que podamos postular

15 Hacemos hincapié en que esto define al problema mente-cuerpo. Para Chalmers, el problema mente-cuerpo es el hard problem of consciousness y el hard problem of consciousness es el problema (del concepto) de la mente fenoménica. 
principios psicofísicos que funden esta nueva metodología no-reductiva; esto es, principios teóricos que permitan conectar “(...) las propiedades de los procesos físicos a las propiedades de la experiencia (...) [, de manera que] encapsularan la manera como la experiencia surge de lo físico (...)" (Chalmers, 1998a: 22). Como bien resume Chalmers:

En última instancia, estos principios deberían decirnos qué clase de sistemas físicos tendrían experiencias asociadas, y para los sistemas que las tienen, ellos deberían decirnos qué clase de propiedades físicas son relevantes para el surgimiento de la experiencia y justamente qué clase de experiencia deberíamos esperar que sea producida por cualquier sistema físico (Chalmers, 1998a: 22).

\section{§3. Dualismo naturalista: crítica y problemas}

Hasta ahora nos hemos dedicado a examinar la propuesta de Chalmers, el dualismo naturalista, frente al problema mente-cuerpo, ya que su postura implica tanto una lectura especifica como la delimitación de un objeto en específico como el origen de tal problema y, por consiguiente, conduce y determina una manera de pensar su superación. La interpretación de Chalmers del problema mente-cuerpo, como vimos, se centra en el sentido fenoménico sensible de la mente o en el hecho de que el procesamiento de información física está acompañado por un estado cualitativo sensible que define a la experiencia consciente, y es a esto a lo que se refiere el hard problem of consciousness. En este contexto, la forma de superar el problema implica elaborar una metodología no-reductiva que, de acuerdo con Chalmers, debe fundarse en principios que permitan conectar los estados cualitativos sensibles que definen a la experiencia consciente. La fundación de estos nuevos principios implica, en consecuencia, asumir al estado cualitativo sensible (también llamado conscious experience o simplemente experience) como una entidad fundamental más. En términos de Chalmers, se trata de"(...) tomar a la experiencia como una característica fundamental del mundo (...)" (Chalmers, 1998a: 20): como extra ingredient ${ }^{17}$. Es a esto a lo que nos referimos con que Chalmers supone una ampliación ontológica.

17 Repetimos la expresión que usa constantemente Chalmers para caracterizar esta ampliación ontológica. 
Partiendo del hecho de que Chalmers intenta dar una respuesta al problema mente-cuerpo, detectamos dos momentos: de un lado, un momento interpretativo del problema y, de otro, una prescripción de cómo superarlo. Asimismo, reconocemos que la superación planteada por Chalmers es coherente con su planteamiento; es decir, si aceptamos los puntos de partida de Chalmers (la distinción entre dos conceptos de la mente, uno psicológico y otro fenoménico, y su caracterización de lo fenoménico sensible o de la experiencia consciente como un estado cualitativo sensible), se sigue que postule que deba haber un ingrediente extra (ampliar la base ontológica de la cual parte la ciencia). Sin embargo, sostenemos que la lectura del problema de la cual parte acarrea ciertos puntos cuestionables; con ello, ponemos en tela de juicio el hecho de que la respuesta de Chalmers al problema mente-cuerpo cumpla con ser, efectivamente, una superación.

Dicho de modo breve, lo que sostenemos como cuestionable son las implicancias ontológicas con las que se compromete el dualismo naturalista propuesto por Chalmers. Pensamos que, por un lado, si se lleva tal postura a sus máximas consecuencias, esta abre la puerta a pensar que el panpsiquismo es seriamente posible ${ }^{18}$. Y, por otro lado, si el problema mente-cuerpo deviene un problema propiamente ontológico (un problema sobre la constitución de la realidad), ello implicaría partir de una caracterización equívoca de la experiencia consciente. Sin embargo, poniendo entre paréntesis el tema de si el problema mente-cuerpo es efectivamente una cuestión ontológica o no, nuestra preocupación con la posibilidad del panpsiquismo radica en que este hace patente el hecho de que lo mental o psíquico pensado en este registro no cumple ningún rol explicativo. Y, por lo tanto, el dualismo naturalista, en tanto es la condición teórica que anima la posibilidad del panpsiquismo, se evidencia como una superación inefectiva del problema mente-cuerpo. En lo que sigue profundizaremos sobre estos puntos.

Como punto de partida, valdría la pena pensar por qué surge en primer lugar el problema mente-cuerpo; es decir, ¿a qué responde preguntarnos

18 Pensamos que la fuerte contra-intuitividad que implica esta tesis debería ser una señal para reevaluar los puntos de partida que la hacen posible. Chalmers, sin embargo, parece pensar todo lo contrario. 
por esta vinculación? Si repasamos esta pregunta desde un enfoque científico, nos damos cuenta de que surge por la búsqueda de explicación de la conducta o el comportamiento humano (lo externo observable). Y, para ello, apelamos a que el ser humano tiene él mismo y para sí una experiencia mental (interna e inobservable) que lo conduce y determina su relación con el mundo; de ahí que para la ciencia sea importante preguntarse por cómo entender la mente. De este modo, la neurociencia, la ciencia cognitiva y la psicología ${ }^{19}$ (principalmente la conductual y cognitiva) intentan cada una dar una explicación a la relación entre estos dos aspectos. Sin embargo, siempre parece haber algo que se escapa: aquella realidad mental en su sentido más intuitivo y próximo a nuestra experiencia del mundo y de nosotros mismos.

Ahora bien, en relación al origen del problema, nos permitimos centrarnos en el enfoque científico, pues es este del cual parte y del que se ocupa Chalmers. Así, es a este enfoque al cual pretende responder desde la filosofía, pero con el objetivo de sentar las bases para elaborar una teoría unificadora que permita dar cuenta de esta vinculación. En este contexto, hablar de una teoría unificadora de la mente implica apuntar a poder darle un rol a la experiencia consciente dentro de la explicación de la conducta, y ese es el papel que pretende desempeñar su dualismo naturalista.

Sin embargo, notamos que el dualismo naturalista, llevado a sus máximas consecuencias, deviene una defensa de la posibilidad del panpsiquismo en tanto tesis ontológica (Chalmers, 2013). Ello es así dado que, en primer lugar, Chalmers parte de una lectura ontológica del problema mente-cuerpo. En este contexto, el problema mente-cuerpo es el problema del vínculo entre dos tipos de propiedades fundamentales (de las cuales se dice que está compuesta la realidad), el tipo físico y el tipo mental (fenomenal sensible), y la manera de vincularlas, siguiendo el esquema de toda explicación científica, es partir de elementos simples de cuya interacción, gobernada por leyes, se puedan explicar los elementos compuestos existentes. De este modo, postular elementos simples significa postular entidades fundamentales

19 Nos referimos a la psicología conductual y cognitiva primordialmente. 
a las que se les atribuyen propiedades fundamentale ${ }^{20}$ y de las cuales se derivan los principios teóricos fundamentales ${ }^{21}$ que permiten sostener y fundar esta conexión. Desde esta lectura ontológica, superar el problema significa añadir un extra ingredient, que es el estado cualitativo que define a la experiencia consciente, sobre el cual hemos discutido anteriormente. En segundo lugar, leer el problema mente-cuerpo como un problema ontológico significa que forma parte de un debate entre dos tesis ontológicas: el materialismo y el dualismo. Según Chalmers, el materialismo, en tanto tesis ontológica, postula que "(...) todo es fundamentalmente físico (...)"; mientras que el dualismo, en tanto tesis otológica, postula que "(...) no todo es fundamentalmente físico y que las cosas que no son fundamentalmente físicas son fundamentalmente mentales (...)" (Chalmers, 2013: 3).

Por su parte, el panpsiquismo, cuya posibilidad defiende Chalmers, sostiene, de manera general, (1) que "(...) todo es (o al menos algunas cosas son) fundamentalmente físicas y fundamentalmente mentales (...)" (Chalmers, 2013: 3), (2) que "(...) algunas entidades fundamentalmente físicas tienen estados mentales (...) [donde] los estados mentales relevantes (para esta caracterización del panpsiquismo) son experiencias conscientes (...)" (Chalmers, 2013: 1) y (3) que"(...) algunas entidades microfísicas son conscientes (...)" (Chalmers, 2013: 7). De esta manera, el panpsiquismo en tanto tesis ontológica, pero entendido en un sentido general, surge como una tesis que toma los puntos fuertes tanto del materialismo como del dualismo y supera los débiles. En este sentido, implica una superación de la disputa anterior y por ello dice Chalmers que debemos considerar su posibilidad seriamente.

Ahora bien, para darnos una idea de las implicancias ontológicas a las que apunta el dualismo naturalista llevado a sus máximas consecuencias, conviene esbozar un esquema de las tesis principales del panpsiquismo. Nuestra intención no es ahondar y entrar en detalle respecto de este debate que abre Chalmers. El esquema ha sido elaborado recogiendo solamente las tesis e ideas principales. Asimismo, cabe resaltar que Chalmers,

20 Este es el lugar que ocupan la propiedad del tipo físico y la propiedad del tipo mental. 
dentro de los tipos que distingue, simpatiza más con dos: aquella a la que denomina "panpsiquismo constitutivo russelliano" y aquella denominada "panprotopsiquismo russelliano constitutivo".

1) Panpsiquismo constitutivo:

Tesis: "la macroexperiencia está (total o parcialmente) fundada (grounded $^{22}$ ) en la microexperiencia" (Chalmers, 2013: 8).

2) Panpsiquismo russelliano:

Tesis: "algunas quididades son propiedades microfenomenales" (Chalmers, 2013: 10), de modo que "las propiedades fenomenales fundamentales desempeñan roles microfísicos fundamentales y subyacen a la estructura microfísica fundamental" (Chalmers, 2013: 9).

3) Panpsiquismo russelliano constitutivo (la variación a la cual se inclina más Chalmers):

Tesis: "En este punto de vista, las propiedades microfenomenales sirven de quididades, desempeñando los roles asociados con propiedades microfísicas y también sirven como los cimientos (grounds) de las propiedades macrofenomenales. Esto es, la microexperiencia constituye a la macroexperiencia a la par que desempeña roles microfísicos" (Chalmers, 2013: 10).

4) Panprotopsiquismo russelliano constitutivo (una segunda variación que también le parece prometedora a Chalmers):

Tesis: "las entidades físicas fundamentales son proto-conscientes" (Chalmers, 2013: 15) donde las "las propiedades protofenomenales son propiedades especiales que no son fenomenales (...) pero que colectivamente constituyen propiedades fenomenales" (Chalmers, 2013: 15), de modo que es la tesis de que "algunas entidades físicas fundamentales tienen propiedades protofenomenales" (Chalmers, 2013: 15).

22 Relación de constitución metafísica: Si A está grounded en B, entonces, las verdades en relación a $\mathrm{A}$ se obtienen completamente de las verdades de $\mathrm{B}$. 
En primera instancia, esta manera de comprender el problema mente-cuerpo y de plantear su superación nos parece cuestionable, puesto que el concepto de estado cualitativo, experiencia consciente o experiencia (la entidad fundamental que la superación del problema exige añadir), así como el de propiedad fenomenal (la propiedad que el estado cualitativo instancia) son conceptos teóricos que no cumplen ningún rol explicativo. Ellos, en realidad, no desempeñan ninguna función en particular a la hora de articular una explicación de cómo se vincula la mente (la dimensión interna e inobservable) con el cuerpo (lo externo y observable) ${ }^{23}$, sino que yacen en el nivel de simples y meros principios teóricos, esto es, en un sentido meramente nominal.

Lo dicho se hace patente en Panpsychism and Panprotopsychism (2013), pero ya desde 1995 aparece con Facing up to the Problem of Consciousness (1995), puesto que luego de distinguir entre el hard problem of cosciousness (la experiencia consciente) y los easy problems (los procesos o funciones mentales), esta distinción no afecta propiamente a la metodología científica actual como pretende Chalmers. Por ejemplo, su principio psicofísico de la coherencia estructural postula simplemente que hay una coherencia entre la estructura de la percatación (awareness) y la de la conciencia (consciousness): “(...) la estructura tridimensional del espacio del color fenomenal (...) corresponde directamente a la estructura tridimensional de la percatación (awareness) visual (...)" (Chalmers, 1996: 23). La percatación (awareness) para Chalmers es un proceso cognitivo; es decir, forma parte del sentido psicológico de la mente y, por lo tanto, es definible funcionalmente, como habíamos ya subrayado en apartados anteriores. Esto quiere decir que la ciencia que investiga la conciencia humana podría seguir empleando su metodología de investigación actual, pero con la única diferencia de que, ahora, tendría el derecho formal de decir que de tales procesos son estructuralmente coherentes con la experiencia consciente acompañante.

Asimismo, en The Conscious Mind (1996) retoma este punto con la agravante de que una vez más reitera que "(...) en la medida en que la conciencia ${ }^{24}$

23 Acá estamos siguiendo la lectura de corte científico sobre el problema que esbozamos momentos anteriores.

24 También denominada por Chalmers como experiencia consciente. 
es el problema realmente difícil de una ciencia de la mente, la conciencia fenoménica ocupará el lugar central (...)" (Chalmers, 1996: 22); pero, dado lo expuesto, esta centralidad parece perderse en la mera mención o en la mera intención. De esta manera, si apelamos al origen del problema mente-cuerpo desde el enfoque científico que ya esbozamos y al objetivo que tiene de superar tal problema, entonces, permanece como una incógnita cuál es el sentido de tales conceptos más allá de sostener que entidades tales como quarks y fotones tienen estados cualitativos o experiencia consciente. Es decir, no es evidente - $y$, de hecho, es demasiado lejano- cómo se articulan a la manera de explicación de la mente humana, la cual, en principio, es deseable por su poder predictivo ${ }^{25}$.

En segundo lugar, tal forma de comprender el problema mente-cuerpo y de plantear su superación nos parece cuestionable, puesto que su punto de partida implica un sentido equívoco acerca de la referencia del concepto de hard problem ${ }^{26}$. El equívoco, para nosotros, consiste en sostener que la experiencia consciente se define por ser un mero estado cualitativo sensible ${ }^{27}$ o sensación cualitativa sensible (qualitative feel, término que aparece en 1996), en lo cual detectamos que los conceptos de experiencia consciente (como estado cualitativo) y de propiedad fenomenal de Chalmers carecen de rol explicativo. La razón de que esto sea así tiene su raíz en el sentido de mente fenoménica, el cual articula, justamente, los dos conceptos anteriores $y$, con ello, determina el sentido del concepto de experiencia consciente al que se suscribe Chalmers. Sin embargo, para nosotros, caracterizar la experiencia consciente (o simplemente experiencia) solamente como algo cualitativo sensible es equívoco, ya que carece del rol o del poder explicativo del concepto mismo.

Para comenzar, Chalmers parte de una serie de equivalencias que no justifica ni en Facing up to the Problem of Consciousness (1995) ni en The Conscious

25 La tesis es que explicar la mente humana es una empresa científica deseable en la medida en que su explicación permite la predicción de comportamientos futuros.

26 Estamos de acuerdo con que existe un hard problem, pero estamos en desacuerdo respecto de cómo se entiende la referencia del concepto de hard problem.

27 Acuñamos este término porque pensamos que reúne lo común a todas las maneras que emplea Chalmers para caracterizarlo; el siguiente término sí es uno de aquellos que usa el mismo Chalmers. 
Mind (1996) y que, por lo tanto, da por sentada. Así, para Chalmers es equivalente hablar de "conciencia fenomenal sensible" (phenomenal consciousness, 199528), "experiencia" (1995, 1996), "experiencia consciente" (conscious experience, 1995 y 1996) "qualia" (1995 y 1996), "sensación cualitativa sensible" (qualitative feel, 1996), "cualidad fenomenal sensible" (phenomenal quality, 1996), "aspecto interno" (internal aspect, 1996), "aspecto subjetivo" (subjetive aspect, 1995) y "cualidad subjetiva de la experiencia" (subjective quality of experience, 1996); puesto que todo ello tiene como referencia (apelando a un ejemplo de Chalmers) a la sensación cualitativa sensible de lo rojo (the qualitative feel of redness, 1995). Es por esta razón que nos hemos permitido referirnos al hard problem como el problema del estado cualitativo.

Asimismo, cuando Chalmers, en diversos lugares, empieza a hablar de este problema, hace una apreciación y una lectura bastante intuitiva de lo que está en juego al hablar de la experiencia consciente, de modo que quien lo lee (o lo escucha) capta de manera innegable cierta verdad en lo que dice. Sin embargo, es cuando vamos a los detalles de sus textos donde aparecen los problemas ${ }^{29}$. Por ejemplo, surge la preocupación respecto de la definición de experiencia consciente, en la medida en que esta parece en parte contra-intuitiva, ya que es un concepto cuya caracterización carece de un concepto de sujeto. Si se la examina de cerca, la caracterización que da Chalmers no contempla ningún tipo de yo (self) o sujeto para el cual este modo sea relativo, vaya dirigido, o sea, para empezar, manifiesto ${ }^{30}$. Este es un punto crucial, dado que este es, justamente, el concepto por el cual se dice que la experiencia consciente remite a un aspecto subjetivo en primer lugar.

28 Los años hacen referencia a los libros en los que aparece el término sin que se remitan a una referencia paginable.

29 Nos referimos no solamente al esquema de interpretación que elabora Chalmers del problema mente-cuerpo y sus dos conceptos de mente, sino también a los ejemplos que usa para sustentar la realidad de su concepto fenoménico de mente (Chalmers, 1996: 6-9).

30 "Sometimes terms such as 'phenomenal consciousness' and even 'qualia' are also used, but it is natural to speak of 'conscious experience' or simply 'experience'. These terms simply imply here that the process being studied (vision, pain, memory, imagination, etc.) appears as relevant and manifest for a 'self' or 'subject' that can provide an account; they have a 'subjective' side" (Varela y Shear, 1999: 1). 
En el marco de la propuesta de Chalmers, si un organismo es consciente, there is something it is like to be ${ }^{31}$ ese organismo, en tanto agente cognitivo (recordemos nuestra discusión previa sobre el principio de coherencia estructural y cómo entiende el awareness). De este modo, “(...) un ser es consciente si existe algo como ser (something it is like) ese ser, para usar la frase que hizo famoso a Thomas Nagel. Similarmente, un estado mental es consciente si existe algo como estar (something it is like) en ese estado mental (...)" (Chalmers, 1996: 4). El problema surge ya que el acento está únicamente en el modo (o el cómo) en el que se da la experiencia, pero pensándolo como una propiedad que se instancia (what it is like to ${ }^{32}$ en un proceso; es decir, sin un someone for whom there is something it is like en primer lugar.

Dado el acento que le pone Chalmers a la sensación cualitativa, el yo (self) presente en la experiencia consciente queda excluido de la ecuación y, en ese sentido, el concepto de conscious experience de Chalmers parece carecer de todo poder explicativo. De esta manera, interpretaciones naturalistas como la de Chalmers, que intentan replicar el esquema de explicación de las ciencias naturales respecto de la conciencia, en realidad sacan a la luz lo que Husserl, por ejemplo, ya señalaba (y frente a lo cual su postura fenomenológica reaccionaba): el hecho de que, al ser “(...) su meta (...) alcanzar la 'pura determinación objetiva' de la realidad 'en sí misma', [termina] poniendo 'fuera de juego' y 'batallando en contra' de todo lo subjetivo (Hua IX, §26) (...)" (Rizo-Patrón, 2016: 65-6). Así pues, lo que habría que pensar seriamente no es tanto la posibilidad del panpsiquismo, sino en todo caso la posibilidad de la fenomenología.

31 Chalmers invoca y usa para sus propios propósitos la expresión célebre de Thomas Nagel (1974).

32 Estamos refiriéndonos a la expresión que emplean Zahavi y Gallagher para identificar la referencia del concepto de experiencia consciente de las que parten posturas como la de Chalmers (Gallagher y Zahavi, 2008: 49). 


\section{$\S 4$. Conclusiones: resumen y reflexiones finales}

Empezamos nuestro artículo sosteniendo que leer el problema mente-cuerpo en clave sujeto-objeto es una manera crucial de superar el problema; sin embargo, el camino que escogimos para hacer valer nuestro punto de vista fue el de una vía negativa. Es decir, partimos del examen de una postura que no consideraba esta dimensión del problema, sino que más bien realizaba una interpretación en clave ontológica del mismo. Esta postura es la de David Chalmers y su lectura ontológica del problema mente-cuerpo descansa, como vimos, en la propuesta del dualismo naturalista.

El dualismo naturalista parte de una naturalización de la conciencia que no considera la lectura sujeto-objeto que nosotros consideramos crucial, y de ahí nuestra intención de discutir la postura de Chalmers: su interpretación del problema en el sentido de su superación. Esto configuró dos objetivos: uno general y uno específico. El primero apuntaba a examinar el carácter ontológico de la postura de Chalmers en torno al problema mente-cuerpo. El segundo planteaba una crítica a lo que postula Chalmers como superación de dicho problema: el hecho de que esta implique añadir un extra ingredient. Esto se hizo desde dos flancos: las implicancias a las que conduce el dualismo naturalista llevado al extremo y los presupuestos del dualismo naturalista que hacen, en última instancia, posibles tales implicancias.

De esta manera, vimos que el problema mente-cuerpo desde la lectura ontológica de Chalmers que naturaliza la conciencia termina por quitarle poder explicativo al mismo concepto de conciencia del que parte. Esta carencia de rol explicativo se hace patente, de un lado, en la posibilidad del panpsiquismo y, de otro lado, en la omisión del sujeto o yo (self) como aquello que constituye la experiencia consciente. $Y$ es justamente por esta razón que sostenemos que la superación del problema mente-cuerpo termina siendo inefectiva.

Ahora bien, dijimos además que la superación del problema en cuestión no debería ni tendría por qué seguir un camino ontológico. No debería, por las implicancias que ya hemos revisado: de un lado, se postula como posible algo, el panpsiquismo, que parece más un sinsentido por la fuerte carga 
contra-intuitiva que lleva consigo sin que termine por dar una respuesta al problema por el cual, en principio, se postula como una seria posibilidad; de otro lado, se quita al sujeto de la ecuación. Y no tendría por qué seguir tal camino en la medida en que existen otras alternativas más provechosas, como son aquellas aproximaciones de corte más fenomenológico.

La tesis de la que partía nuestra crítica afirmaba que la superación del hiato mente-cuerpo se encuentra en leer el problema como el de la conciliación entre el sujeto y el objeto, en la medida en que el primero se considera el agente que constituye al segundo. De esta manera, como señala Varela, desde la misma ciencia contemporánea al problema “(...) la aproximación fenomenológica parte de la irreductibilidad de la naturaleza de la experiencia consciente (...)" (1998: 341) y, por consiguiente, pone el acento en la experiencia misma en la medida en que su estructura es el único vínculo entre la mente y la conciencia. En ese sentido, afirma también Shear junto a Varela "(...) la experiencia vivida es donde empezamos y a donde todo debe conducirnos de regreso, como una cuerda guía (...)" (Varela y Shear, 1999: 1), si es que la superación del problema mente-cuerpo ha de tener algún sentido real. Por ello, ambos apuntan en primera instancia a un camino menos pretencioso, a un cambio en la manera como comprendemos lo que está en juego en el problema mismo.

Nos acogemos entonces a una lectura más cercana a la fenomenología para intentar superar el hiato, pero esto que decimos de una manera aparentemente inocente e inofensiva acarrea nuevos puntos que ameritarían ser discutidos con mayor profundidad. En lo que sigue, solo esbozaremos un par de reflexiones finales al respecto.

En primer lugar, cuando planteamos que suscribirnos a una lectura más fenomenológica del problema podría cumplir efectivamente con superarlo, tenemos en mente, en buena cuenta, a la fenomenología husserliana. Esto se debe a que, en la medida en que está bajo el lema de "ir hacia las cosas mismas", no parte ni “(...) consiste en la presentación y discusión 'taxonómica' de distintas alternativas -metafísicas y epistemológicas (monismos, dualismos, funcionalismos, eliminativismos, reductivismos o no, etc.)-, con sus respectivas categorías causales, funcionales u otras (...)" (Rizo-Patrón, 2016: 
63) y, gracias a ello, su concepto de conciencia sí cumple un rol explicativo. Asimismo, también pensamos en perspectivas más contemporáneas, tanto científicas como filosóficas, que buscan reactualizar las aproximaciones fenomenológicas tanto de Husserl como también las de Merleau-Ponty.

Estas aproximaciones las encontramos, por ejemplo, en Zahavi y Gallagher (2008) o en Varela, quienes, desde la neuro-fenomenología, parten de leer el problema como el de la perspectiva de primera y tercera personas. $Y$ si bien en cierto sentido podrían no ser suficientes (dado el concepto de conciencia con el que parten), parecen caminos más fructíferos que el de Chalmers, pues parten de reconocer que

“(...) hay más [en lo concerniente] a la experiencia que el hecho de que lo que es percibir (the fact that what it is like to perceive) un triángulo negro es subjetivamente distinto de lo que es percibir (what it is like to perceive) un círculo rojo (cf. Nagel, 1974). No solo es lo que es percibir (what it is like to perceive) rojo diferente de lo que es recordar o imaginar (what it is like to remember or imagine) rojo. Además, todas estas experiencias fenomenales implican una referencia a un sujeto de experiencia. Al percibir o imaginar un objeto conscientemente, uno es consciente de que el objeto aparece de manera determinada para uno mismo. (...)" (Gallagher y Zahavi, 2008: 49).

Y, por lo tanto, sus planteamientos ofrecen, al menos, un mayor poder explicativo.

\section{Bibliografía}

Chalmers, David, 1995. Absent Qualia, Fading Qualia, Dancing Qualia. En: Conscious Experience, ed. Thomas Metzinger. Paderborn: Schöningh/Imprint Academic, 309-328.

- 1996. The Conscious Mind: In Search of a Theory of Conscious Experience. ftp://121.17.126.74/data1/ts01/english/novel/batch001/201005112100161474. pdf. Consultado 15 de octubre de 2017.

- 1998a. Facing Up to the Problem of Consciousness. En: Explaining Consciousness: The Hard Problem, ed. Jonathan Shear. Londres: The MIT Press, 9-31. 
- 1998b. Moving Forward on the Problem of Consciousness. En: Explaining Consciousness: The Hard Problem, ed. Jonathan Shear. Londres: The MIT Press, 379-422.

- 1999. La mente consciente: en busca de una teoría fundamental. Traducción de José A. Álvarez. Barcelona: Gedisa.

- 2013. Panpsychism and Panprotopsychism. The Amherst Lecture in Philosophy 8, 1-35. http://www.amherstlecture.org/chalmers2013/. Consultado 15 de octubre de 2017.

Gallagher, Shaun y Dan Zahavi, 2008. The Phenomenological Mind: An Introduction to Philosophy of Mind and Cognitive Science. Nueva York: Routledge.

Rizo-Patrón de Lerner, Rosemary, 2016. La crítica trascendental al naturalismo. Actualidad de la aproximación fenomenológica husserliana. Enrahondar. An International Journal of Theoretical and Practical Reason 57, 53-77.

Varela, Francisco, 1998. Neurophenomenology: A Methodological Remedy for the Hard Problem. En: Explaining Consciousness: The Hard Problem, ed. Jonathan Shear. Londres: The MIT Press, 337-357.

Varela, Francisco y Jonathan Shear, 1999. First-Person Methodologies: What, Why, How? En: The View from Within: First-Person Approaches to the Study of Consciousness, eds. Jonathan Shear y Francisco Varela. Exeter: Imprint Academic, 1-14. 